\title{
Issues of regulation of fillability of a train traffic schedule
}

\author{
Sergey Bessonenko, ${ }^{1, *}$, and Mikhail Badazhkov ${ }^{1}$ \\ ${ }^{1}$ Siberian Transport University, Dusi Kovalchuk st., 191, 630049, Novosibirsk, Russia
}

\begin{abstract}
Fillability of a schedule, down time of trains, quality of a schedule, the use of train paths, reduction of operating costs, train paths, distribution of trains. The problem of reduction of operating costs for the train traffic between technical stations is considered. The analysis of the use of a train schedule is made in this paper. The dependence of operating costs on the route speed, the quality of a schedule and its fillability is established. A solution to the problem of improving the quality of filling the schedule, expressed in the use of train paths of certain categories (direct, or having a stop on the section between the technical stations) is proposed. A method for determining the down time of trains at technical stations is proposed, depending on the fillability of a schedule. The forecast of the change in the main operating indicators and operating costs for the application of the proposed technology for the departure of trains was made.
\end{abstract}

\section{Introduction}

The problem of making the optimal schedule for the train traffic, which makes it possible to link the work of all the railway units, is on the agenda throughout the entire existence of the railway transport. Today, the problem of the economic efficiency of the train traffic schedule is of a great importance. One of the examples of realization of the idea of economically optimal schedule is the "Elbrus" system. However, there are a number of unsolved problems in the field of schedule optimization at the moment.

\section{Increasing the speed of trains will significantly reduce operating costs}

The quality of the trains passing through the section depends on the schedule [1]. The schedule, fillability of the schedule and percentage of performance vary depending on the period of the year, and depending on the operating domain under consideration [2]. To study the performance indicators for the train traffic schedule, the methods of mathematical statistics are usually used [3].

\footnotetext{
*Corresponding author: bessonenkosa@mail.ru
} 
On the example of one of the heavy traffic sections of the network, data on down time of trains at technical stations, route speed, and quality of the schedule performance for the departure and passing of trains were analyzed.

Figure 1 shows the relationship between operating costs and route speed.

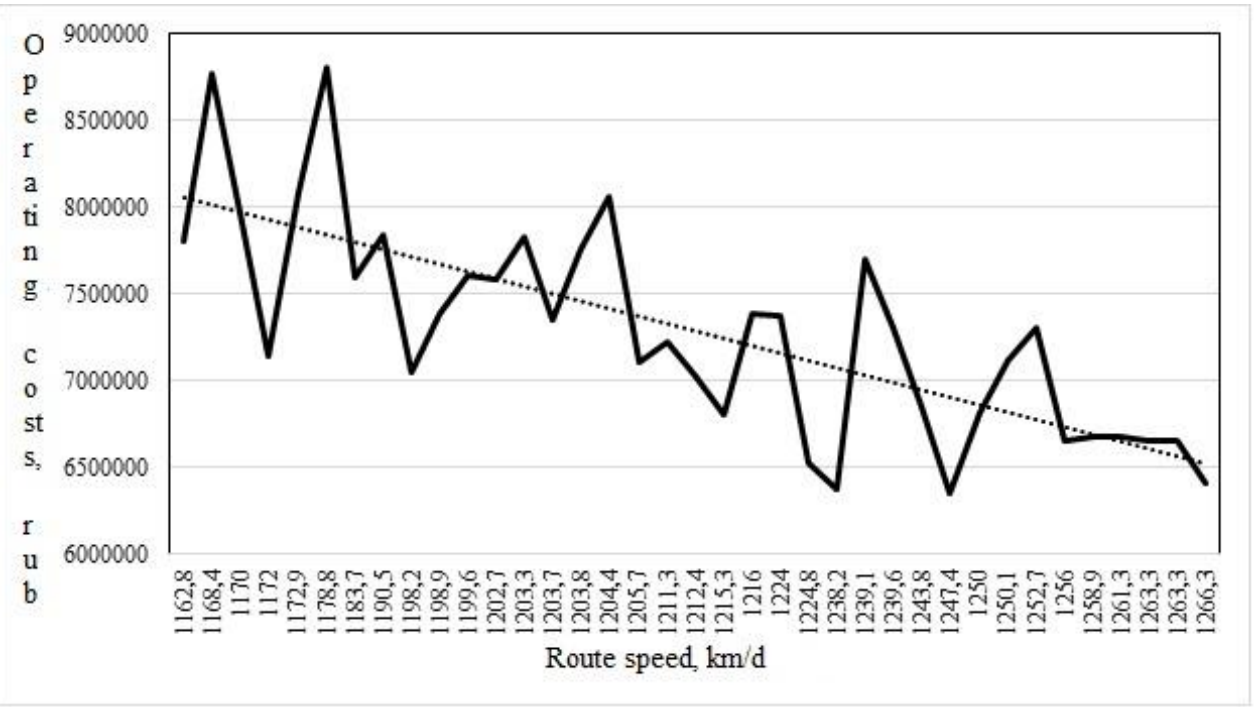

Fig. 1. The graph of the dependence of operating costs on route speed.

Based on the analysis of the schedule of changes in costs and route speed, we can conclude that they are inversely proportional. The dependence is not linear, since other factors influence the change in operating costs, except for the route speed. On the basis of the data obtained, it can be concluded that the increase in route speed leads to a reduction in costs. It can be seen from the graph above that when the route speed is increased by $8.6 \%$ of the minimum value, the operating costs are reduced by $17 \%$.

The decision which allows reducing costs is the increase of the route speed, and, accordingly, the improvement of the quality of performing and scheduling of train traffic. Increasing the route speed by $10 \mathrm{~km} /$ day, one can achieve a reduction in operating costs by $1.7 \%$. The route speed depends on the service speed and time of stop at service stations.

The service speed, in turn, depends on the technical speed and also on the number and duration of stop at the intermediate stations of the section for the handling of trains that have bigger advantage (usually passenger ones). Aspiration for maximum service speed as a target indicator does not guarantee minimization of cash costs for operational work [4].

The following parameters directly depend on the quality of scheduling the train traffic:

- time of stop at service stations,

- the number and duration of stop at intermediate stations,

- technical speed.

From the economic point of view (as a rule, with small traffic sizes), it is not always rational to use the train paths, which have stops at intermediate stations of a section for the handling of passenger trains, in view of the emerging additional costs. The stops on a section are associated with additional costs for the acceleration / deceleration of trains, the operating time of locomotive crews, the turnover of locomotives (locomotives and locomotive crews are in actual operation only about half of allocated time $[5,6])$, and on the power consumption directly during the stop. Stops often lead to a deviation from the schedule. There are two ways to reduce the operating costs associated with the train traffic. 
1. Development of the "most economical" version of the schedule, i.e. depending on the volume of traffic, an optimal balance of following parameters should be reached in this schedule:

- time of stop at service stations;

- stops at intermediate stations;

- number of train paths;

- technical speed.

2. Effective and most rational use of the existing train traffic schedule, taking into account the aspiration to balance the parameters.

The second variant is considered in this paper. As a basis for the rational use of the train paths, a flexible system for regulating the fillability of the train traffic schedule on a section limited by service stations has been adopted.

\section{Impact of schedule fillability on the down time of a car at service station}

Fillability of a train traffic schedule and daily down time of transit trains are considered.

This analysis was carried out to determine the relationship between the fillability of a schedule and the down time of trains at service stations. It is established that as the fillability of a schedule increases, the amount of down time of trains, although tends to increase in general, but still varies randomly. One of the explanations for this change in down time is the limited number of data. On the other hand, besides the train paths, there are many other reasons which impact on the down time of trains: lack of locomotives, uncoupling of bad-order cars, technological "windows", etc. These reasons must be taken into account. As part of this analysis, it was revealed that during the period under consideration the above-mentioned reasons seriously affected the worsening of down time. On the basis of the schedule of the performed train traffic, cases of exceeded down time of trains for reasons not depending on the quality of the scheduling are revealed and down time is adjusted. Graphs of the dependence of down time of trains on the percentage of the use of train paths are made, taking into account the adjustment of down time, and shown in Fig. 2, 3 .

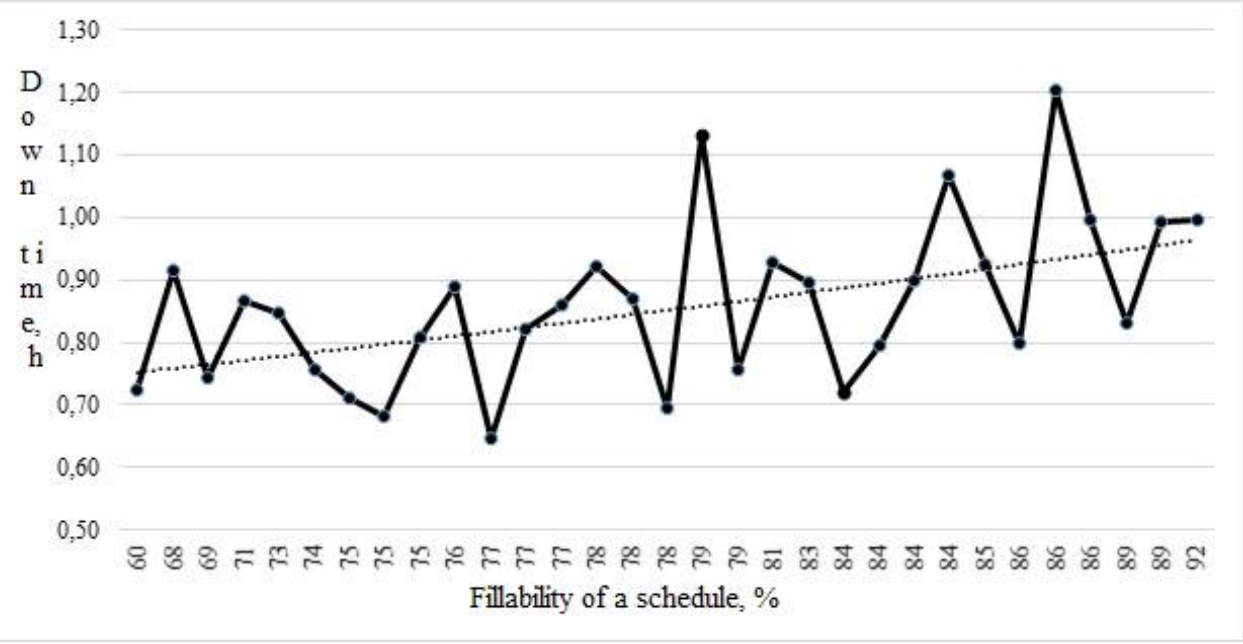

Fig. 2. Graph of dependence of down time on the fillability of a schedule on the odd side. 
As one can see, the percentage of fillability of a schedule can vary in a wide range of values. The trend line on the graphs indicates an increase in down time as the fillability increases. Therefore, it clearly shows the dependence of down time of trains at service stations on the fillability of a schedule.

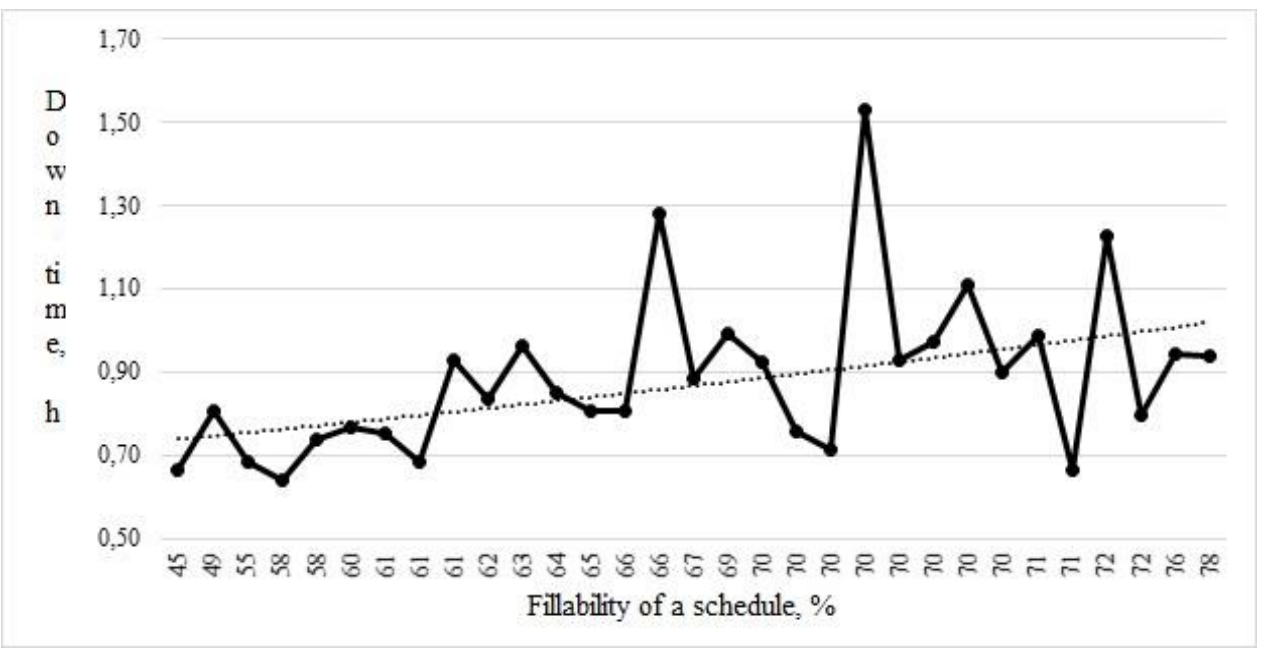

Fig. 3. Graph of dependence of down time on the fillability of a schedule on the even side.

At the same time, if we consider the change in down time along the trend line in the segment from $60 \%$ to $80 \%$, the down time changes from 0.74 hours to 0.88 hours on the odd side in this segment. On the even side - from 0.74 hours to 1.00 hours.

When considering the schedule, it is established that, in addition to the fillability per se, the interaction of parameters such as the standard processing time of the train and the location of the train paths corresponding to it influence the down time. That is, in order to minimize down time, it is necessary that the train paths of departure are located relative to the train paths of arrival in a time interval equal to the train processing time. The correspondence of the number of arrival and departure train paths on the time intervals equal to the train processing time is very important. In the case where the number of the train paths of arrival is less than the number of the train paths of departure, the trains which don't have the train paths will be down in their expectation. Basically, such situations occur when the train paths are "taken down" by passenger trains, when two or more passenger trains are handled for a short time. And if, on average, during the day the number of the train paths of arrival corresponds to the number of the train paths of departure, then this rule does not work within 1, 2 and 3 hour intervals.

To determine the real dependence, the daily normative graph was divided into time intervals equal to the train processing time (for convenience of calculation, it is assumed equal to 30 minutes). The number of train paths of arrival and departure on these time intervals is counted. The number of train paths of arrival in the first time interval is related to the number of train paths of arrival in the second time interval and so on. The time which exceeds the technological norm of down time of trains at the station $t_{\text {ex.down }}$ is calculated according to the following rule. This time is a multiple of the number of trains that stood at the station in excess of the technological norm of time and time of non-regulatory down time. The number of trains corresponded to the difference between the number of train paths of arrival and departure upon condition of the prevalence of the first, including the cumulative total.

$$
t_{\text {ex.down }}=\sum_{i=1}^{48}\left(N_{i} \cdot t_{\text {stand }} / 60\right)
$$


in case of $N_{i} \geq 0$.

Where: $t_{\text {stand }}$ - the standard processing time of the train at the station is assumed to be equal to 35 minutes; $N_{i}$ - the number of trains which stood at the station in excess of the technological time norm of the trains in the time interval $i ; i$ - the serial number of the time interval equal to $t_{\text {stand. }}$.

The number of trains which stood at the station in excess of the technological norm of the time is determined by the formula:

$$
N_{i}=\sum_{j=1}^{i}\left(N_{\mathrm{ar} j} \cdot Z\right)-\sum_{j=1}^{i} N_{\mathrm{dep} j}
$$

where $N_{\mathrm{ar} j}$ - number of train paths of arrival on the section $j ; N_{\mathrm{dep} j+1}$ - number of train paths of departure on the section $j+1 ; Z$ - fillability of a schedule, in shares.

The excess of the train down time norm was determined for the fillability of the schedule from 0.5 to 1 , in increments of 0.05 . After that, the calculation of the total down time of trains covered in the standard traffic schedule was made. The general down time $t_{\text {down }}$ consists of the sum of the standard time for processing the train and the time exceeding it in anticipation of the train path of the schedule and is determined by the formula:

$$
t_{\text {down }}=\frac{\left(\left(\mathrm{N}_{\mathrm{dep}} \cdot Z \cdot t_{\mathrm{stand}} / 60\right)+t_{\mathrm{ex} \cdot \mathrm{down}}\right)}{N_{\mathrm{dep}} \cdot Z}
$$

Obtained values are presented in the form of a graph in Figure 4.

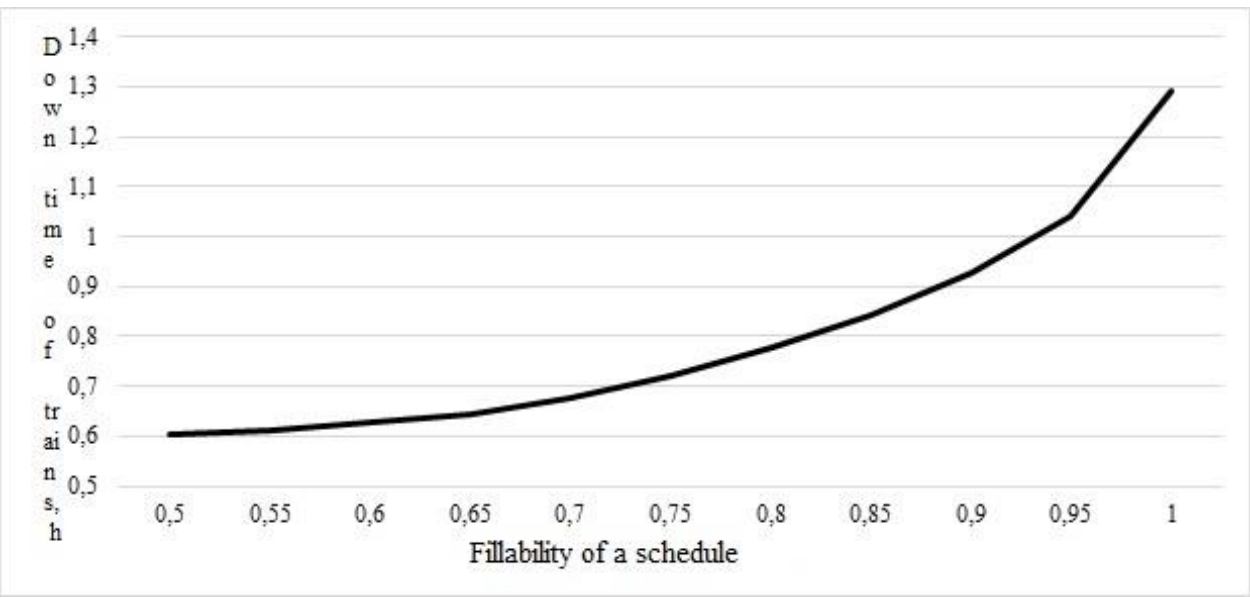

Fig. 4. Graph of dependence of down time of trains on the fillability of the standard schedule.

The exponential dependence of the down time on the fillability is clearly visible on the obtained graph. Comparison of trend lines of graphs obtained empirically and the graph shown in Fig. 4 shows their correspondence to each other. Firstly, the fact that there is no strict exponential dependence on the first graphs is explained by the presence of errors that occur in the performed schedule. Secondly, fast growth of down time occurs only at more than $95 \%$ of the fillability of a schedule, which has not been achieved in practice.

The following conclusions can be drawn from the analysis:

- down time has an exponential dependence on the fillability of the schedule;

- there is a limit to the fillability of the schedule. When it reached, fast growth of the down time of trains occurs.

\section{The need to take into account the weight of trains sent to achieve the economic efficiency of the traffic schedule}


It was previously determined that the schedule is used by about $70 \%$, i.e. it has a $30 \%$ reserve of traffic capacity. With such a reserve, the question arises of the "profitability" of using train paths that have stops at intermediate stations. The question of the quality of the use of the schedule is in the standard quantity and use of train paths that have stops on the section. The energy input from using the train paths directly depends on the weight of the train. Often, the use of the same train path can be reasonable for trains weighing 1500 tons and, at the same time, lead to losses when handling a heavier train. During the analysis, all trains, depending on the weight, were divided into six categories: up to 2000 tons; from 2000 to 3000 tons; from 3000 to 4000 tons; from 4000 to 5000 tons; from 5000 to 6000 tons; from 6000 to 7000 tons; more than 7000 tons. The train paths formed three groups: straight train paths, train paths that have one stop on the section, train paths that have two or more stops. The analysis was carried out separately for the even and odd directions. The diagrams of the percentage distribution of trains between the train paths of different categories, depending on the weight of the trains for the even and odd directions, are presented in Figures 5 and 6.

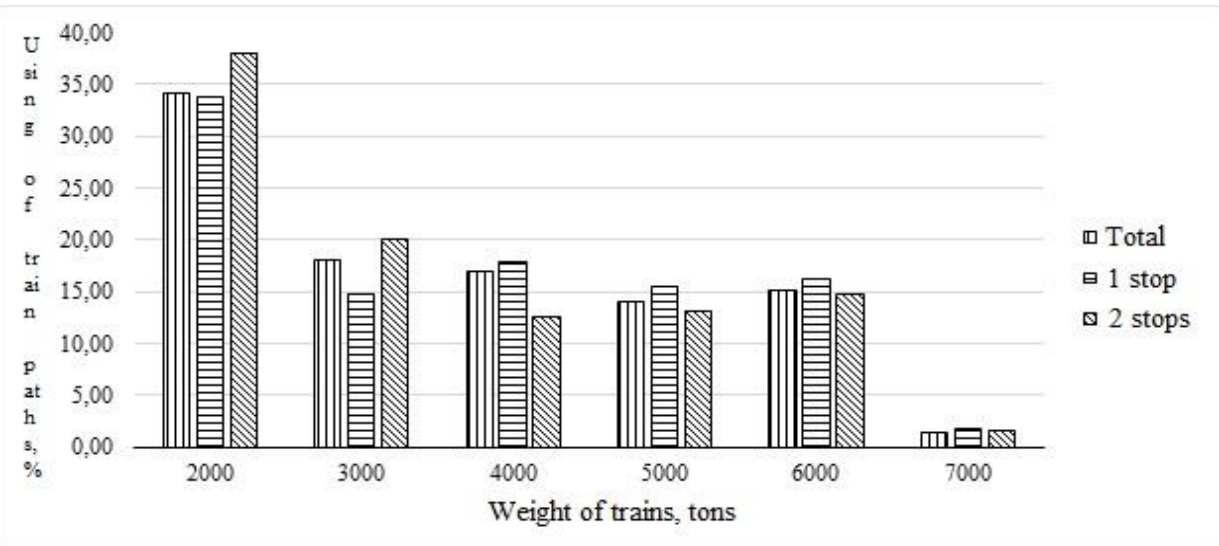

Fig. 5. The diagram of the distribution of even trains between the train paths.

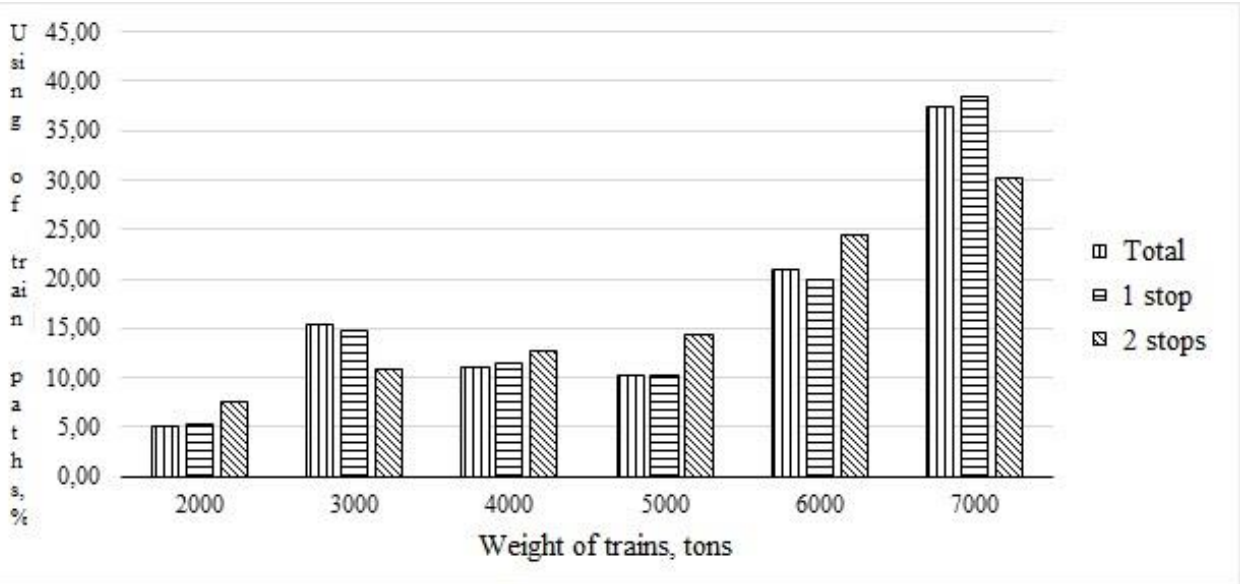

Fig. 6. The diagram of the distribution of odd trains between the train paths. 
In the diagrams presented, it is possible to track the train paths on which trains of different categories went. Generally, different train paths are equally used by trains of different categories. The fluctuation is not more than $9 \%$. This situation is very predictable in circumstances where the weight of the train is not taken into account when planning the train path of departure; all common indicators tend to the average value.

Determination of the proportion of trains sent to the train paths having one, and also two or more stops along the section is of great importance for further analysis. It is necessary to determine the value of this proportion and consider the possibility of a partial or complete refusal to use such train paths, taking into account possible negative consequences.

The proportion of trains having two or more stops in the section was $10 \%$ for even and $6 \%$ for odd directions. $30 \%$ and $43 \%$ of trains were sent to train paths with one stop, respectively. The rest of the trains proceeded the section without stopping. On the basis of the data obtained, two conclusions are obvious:

- train paths having one stop are an important and integral part of the traffic capacity, it is not possible to completely abandon their use;

- traffic volumes using train paths with two or more stops are not significant in general and do not exceed $10 \%$, there is a potential opportunity not to use these train paths to reduce energy losses.

Based on this, it can be affirmed that existing straight train paths are not always used effectively. The use of train paths of different categories is uneven; the proportion of using train paths that have stops on the section relative to the overall fillability of the schedule is higher by $10-20 \%$.

The financial component of the consequences of using different train paths is determined, i.e. the associated operating costs. This paper doesn't have a target to accurately determine the monetary loss, so the costs from energy and other losses are rounded. Figures 7,8 show the graphs of the change in costs depending on the weights of trains and the use of train paths of different categories for even and odd directions.

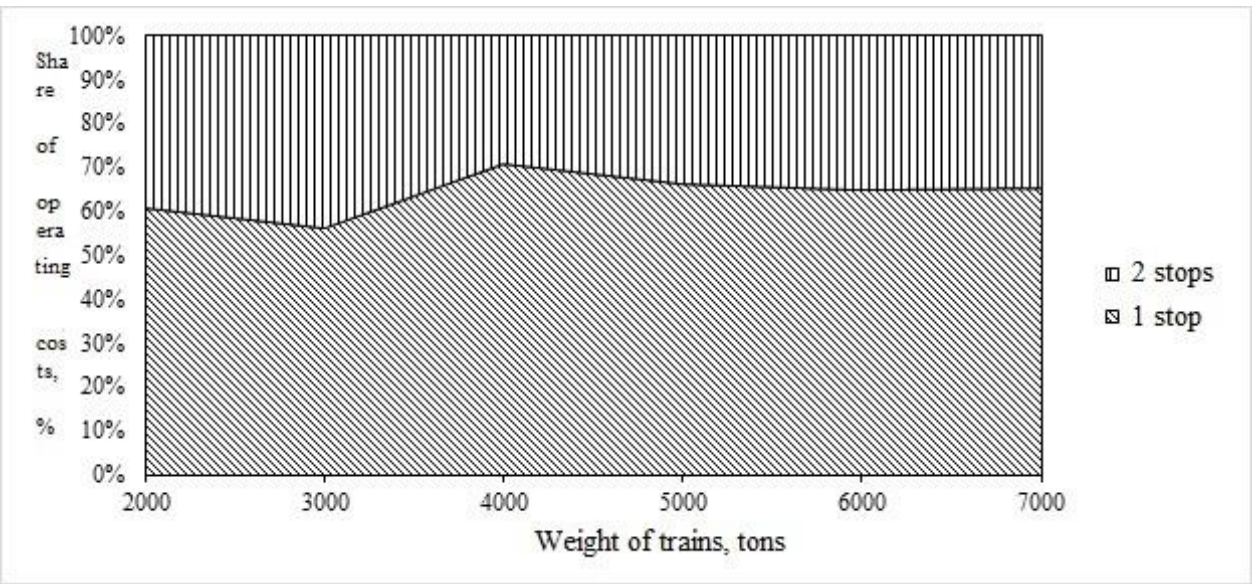

Fig. 7. Graph of changes in costs for the even direction.

The graphs clearly show the ratio of costs occurred when using different train paths. As a result, $10 \%$ of trains of even direction with two or more stops account for $35 \%$ of costs. $6 \%$ of trains of odd direction $-25 \%$ of costs. 


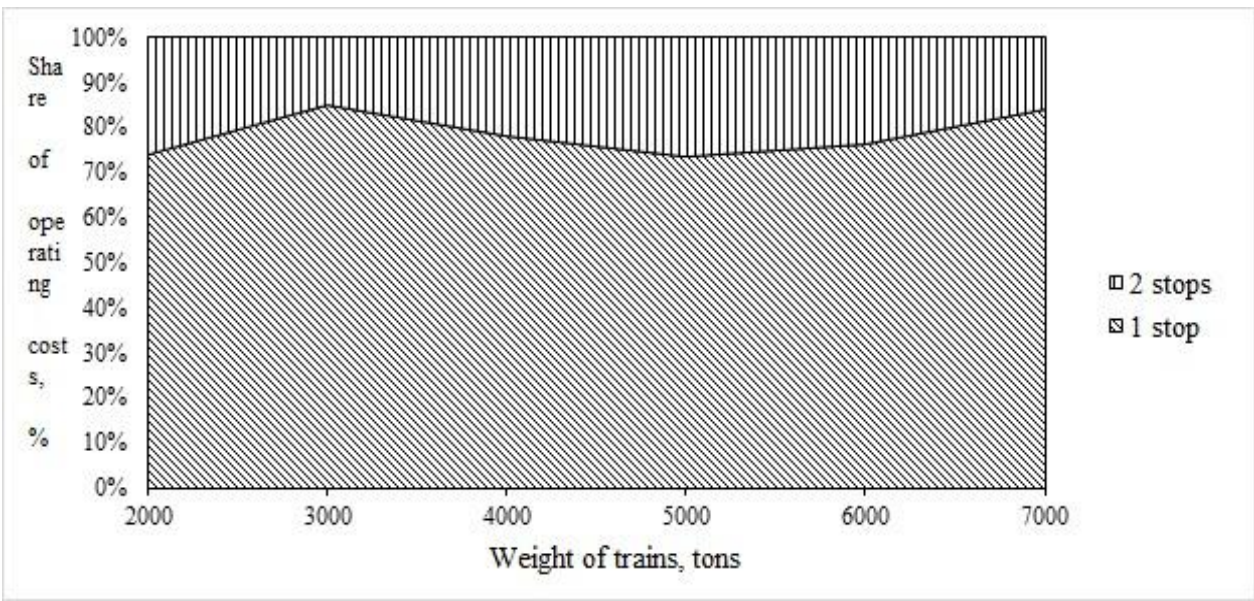

Fig. 8. Graph of changes in costs for the odd direction.

The data given above make it possible to predict the situation of the refusal to use train paths with two or more stops, instead of them straight train paths will be used. Possible increases in the proportion and use of the train paths of normative graph are summarized in Table 1.

Table 1. The proportion of the use of the train paths of normative graph.

\begin{tabular}{|c|c|c|c|c|}
\hline & \multicolumn{2}{|c|}{ Even direction } & \multicolumn{2}{c|}{ Odd direction } \\
\cline { 2 - 5 } & $\begin{array}{c}\text { Performed } \\
\text { schedule, \% }\end{array}$ & Forecast, \% & $\begin{array}{c}\text { Performed schedule, } \\
\%\end{array}$ & Forecast, \% \\
\hline Straight train paths & 71.41 & 83.21 & 62.22 & 69.57 \\
\hline With 1 stop & 72.54 & 72.54 & 98.51 & 98.51 \\
\hline With 2 and more stops & 84.40 & - & 127.96 & - \\
\hline Total & 72.86 & 79.64 & 76.85 & 79.72 \\
\hline
\end{tabular}

As a result of the refusal to use train paths with two or more stops, the percentage of fillability of the schedule increased by $7 \%$ and $3 \%$ respectively for the even and odd side, which on the average corresponds to $5 \%$. The reserve of straight train paths is sufficient for provision of the existing dimensions of traffic without using train paths with two or more stops. However, with such an increase in the percentage of using traffic capacity, the following negative consequences associated with increasing the down time of trains at service stations are occurred:

- increase in the time of use of the receiving and departure tracks;

- increase in the turnover of the locomotive;

- the need to increase the number of receiving and departure tracks of service stations for the possibility of an appropriate decoupling of the schedule.

All these consequences are associated with a corresponding increase in operating costs.

The projected increase in operating costs is calculated on the basis of the trend increase in train down time, depending on the increase in the percentage of use of the train paths, by the method described earlier. Down time of trains will increase by $0.10-0.15$ hours, which corresponds to an increase in expenses by 630 thousand rubles per month. The savings from the reduction of stops for the even and odd sides were given separately above and in total correspond to 1408 thousand rubles per month.

The total savings for one service station is 778 thousand rubles per month or about 10 million rubles per year. 


\section{Increasing operational indicators}

Earlier, the possibility of refusing to use economically unprofitable train paths depending on the weight of trains and the characteristics of the current train situation was substantiated. Availability of traffic capacity reserves is shown. The technology of optimal use of the train paths consists in an individual examination of the possibility of using each train path. The idea of optimization is reduced to solving two main tasks:

1. Determine the reasonability of using a train path (based on the actual number of trains coming to the departure station).

2 . Find the most rational variant of the distribution of trains between the train paths.

Using the proposed technology, the main operational indicators of the railway operation will undoubtedly serve as the efficiency markers. It is with the change in the established indicators of both quantitative and qualitative improvements that we can speak about the efficiency of the proposed technology. Let's consider changing the following performance indicators:

- service speed;

- operating time of locomotive crews.

Service speed is one of the most important operational indicators on which also depends the second indicator of the above list - the operating time of locomotive crews. The calculation of the change in the service speed as a result of the application of the developed technology is made. We will compare it with the indicators factored in normative graph. The calculation is carried out under the following conditions:

- the use of train paths with two or more stops on the section is completely excluded;

- the use of such a number of train paths with one stop is excluded, as a result of which the percentage of using straight train paths will be $90 \%$. The remaining $10 \%$ are left as a reserve in case of failures and breakdowns.

The calculations are carried out according to the formula:

$$
V_{\mathrm{sec}}=\frac{6000 \cdot S_{\mathrm{sec}}}{\gamma_{0} \cdot t_{0}+\gamma_{1} \cdot t_{1}+\gamma_{2} \cdot t_{2}}
$$

where $S_{\mathrm{sec}}$ - the length of the considered section between the service stations, km; $\gamma_{0}, \gamma_{1}, \gamma_{2}$ - the proportion of trains having 0,1 , two or more stops on the section, $\% ; t_{0}, t_{1}, t_{2}$ - the average time required for the passing of the section for train paths with $0,1,2$ stops, min.

After reducing the use of train paths with low energy efficiency, the parameters of the schedule will be calculated according to the formulas given below. Thus, the proportion of using train paths with one stop after the application of the new technology $\delta_{\mathrm{n} 1}$ is (hereinafter, the index $n$ denotes new parameters obtained as a result of removal of inefficient train paths):

$$
\delta_{n 1}=\frac{x_{n 0}+x_{n 1}}{y_{0}+y_{n 1}}
$$

where $x_{n 0}$ - number of trains sent to straight train paths after redistribution of train paths; $x_{n 1}$ - number of trains sent to train paths with one stop on the section; $y_{0}$ - the normative number of straight train paths (const); $y_{n 1}$ - the normative number of train paths with one stop on the section.

$$
x_{n 1}=x-\delta_{n 0} \cdot y_{0}
$$

where $x$ - number of trains sent (const); $\delta_{n 0}$ - the proportion of using straight train paths is assumed to be $90 \%$.

$$
x=x_{0}+x_{1}+x_{2}
$$


Accordingly, the number of trains sent to the straight train paths with one, two or more stops is calculated:

$$
x_{0}=\delta \cdot y_{0}, x_{1}=\delta \cdot y_{1}, x_{2}=\delta \cdot y_{2}
$$

In the calculations, we take the total number of train paths of the normative graph $y$ equal to 1, and then the number of straight train paths with one, two or more stops will correspond to their relative proportions in the normative graph for odd and even directions.

$$
\begin{aligned}
& y_{0(\mathrm{e})}=0,6091, y_{1(\mathrm{e})}=0,3057, y_{2(\mathrm{e})}=0,0851 . \\
& y_{0(\mathrm{o})}=0,6260, y_{1(\mathrm{o})}=0,3381, y_{2(\mathrm{o})}=0,0359 .
\end{aligned}
$$

Under these conditions $x=\delta$, where $\delta$ - fillability of a schedule.

Necessary parameters for calculating the service speed, changed as a result of optimization of the graph, $\gamma_{n 0}, \gamma_{n 1}$ are calculated by the formulas:

$$
\begin{aligned}
& \gamma_{n 0}=\frac{x_{n 0}}{x}=\frac{\delta_{n 0} \cdot y_{0}}{\delta} \\
& \gamma_{n 1}=\frac{x_{n 1}}{x}=\frac{\delta-\delta_{n 0} \cdot y_{0}}{\delta}
\end{aligned}
$$

The overall fillability of the schedule as a result of the changes will correspond to:

$$
\delta_{n}=\frac{x}{y_{n}}=\frac{x}{y_{0}+y_{n 1}}=\frac{x}{y_{0}+\left(y_{1}-x_{n 0}+x_{0}+x_{2}\right)}
$$

For one of the heavy traffic sections, the following results can be given.

The service speed for the even and odd directions factored in the normative traffic graph corresponds to:

$V_{\mathrm{sec}(\mathrm{e})}=59,82 \mathrm{~km} / \mathrm{h} ., V_{\mathrm{sec}(\mathrm{o})}=60,70 \mathrm{~km} / \mathrm{h}$.

Fillability of the graph: $\delta_{\mathrm{e}}=65 \%, \delta_{\mathrm{o}}=78 \%$.

Reducing the proportion of use of train paths with stops on the section, the performed traffic schedule will have the following characteristics:

Fillability of the graph: $\delta_{\mathrm{e}}=79,48 \%, \delta_{\mathrm{o}}=85,53 \%$.

Service speed $V_{\sec (\mathrm{e})}=61,79 \mathrm{~km} / \mathrm{h}, V_{\sec (\mathrm{o})}=61,54 \mathrm{~km} / \mathrm{h}$.

As a result of the increase in service speed, the operating time of locomotive crews is reduced. On average, the operating time for one crew will decrease in the even and odd directions by 0.167 hours and 0.070 hours, respectively.

\section{Conclusion}

1. Insufficiently effective use of the existing train traffic schedule leads to an increase in operating costs.

2. The exponential nature of the relationship between the down time of trains at service stations and the fillability of the schedule is determined. The need to take into account the weight of the train when selecting the train path of departure is indicated.

3. Refusal to use train paths with stops on the section will lead to a serious reduction in operating costs.

4. The decision to use the train path should be made taking into account the weight characteristics of the train.

5. Regulation of fillability of the train traffic schedule on the basis of a balance between time of stops at service and intermediate stations, the number of train paths used will lead to improvement of quality indicators (increase in the service speed, reduction of the operating time of locomotive crews) and reduction of operating costs (primarily from energy losses). 


\section{References}

1. V.N. Tolmachev, Operative management of the handling of trains on double-track sections during periods of repair and engineering works: the author's abstract. Thesis On the Acquisition of the Scientific Degree PhD in Technical Sciences (Moscow, 2004)

2. A.F. Borodin, Bulletin of the Joint Scientific Council of JSC Russian Railways 6, 3-21 (2011) ISSN 2304-9642

3. A.I. Verlan, Vestnik of Dnipropetrovsk National University of Railway Transport 1(49), 75-85 (2014) ISSN 2307-3489 (Print), ISSN 2307-6666 (Online)

4. V.F. Bukhteev, Problems of safety of the Russian society 2, 53-57 (2015) ISSN 23074396 (Print), ISSN 2307-4418 (Online)

5. V.I. Nekrashevich, Vestnik VNIIZhT 4, 8-15 (2006) ISSN 2223-9731

6. G.M. Bilenko, V.S. Volkov, Science and technology of transport 2, 79-86 (2016) 